\title{
Proposta de manejo de Araucaria angustifolia utilizando o quociente de Liocourt e análise de incremento, em propriedade rural no Município de Lages, SC
}

\author{
André Felipe Hess ${ }^{1}$, Andressa Roberta Calgarotto ${ }^{1}$, Renato Pinheiro ${ }^{1}$, Thayana Cristina Rebello Wanginiak ${ }^{1}$
}

${ }^{1}$ Universidade do Estado de Santa Catarina - UDESC, Departamento de Engenharia Florestal, Avenida Luiz de Camões, 2090, CEP 88520000, Lages, SC, Brasil, hessandre@yahoo.com.br; andressarobertac@hotmail.com; alceurpinheiro@yahoo.com.br; thay_31@hotmail.com

\begin{abstract}
Resumo - O objetivo deste trabalho foi calcular o quociente de Liocourt e avaliar a distribuição diamétrica em floresta natural com 84 hectares de Araucaria angustifolia, servindo de parâmetro para o seu manejo em propriedade rural com 176 hectares, localizada na Fazenda Pedras Brancas, Lages, SC, visando atingir o conceito de floresta balanceada. A distribuição diamétrica comportou-se como o esperado para florestas nativas, gerando uma curva de distribuição em J-invertido, demonstrando tratar-se de uma floresta não balanceada em fase de sucessão, por apresentar déficit de árvores nas maiores classes diamétricas. O valor do quociente de Liocourt foi de 1,33, semelhante a outros estudos com florestas naturais. Através da utilização do quociente de Liocourt, verifica-se a possibilidade de retirada de 54 árvores por hectare no conjunto das classes de frequência, para um diâmetro máximo desejado de $35 \mathrm{~cm}$ com redução de $7,4 \mathrm{~m}^{2}$ ha-1 de área basal ou $620,8 \mathrm{~m}^{2}$ para a floresta, evitando-se assim a estagnação, e 39 árvores por hectare para o diâmetro máximo de $40 \mathrm{~cm}$ com redução de $5,27 \mathrm{~m}^{2}$ ha $^{-1}$ de área basal ou $442,7 \mathrm{~m}^{2}$ para floresta.
\end{abstract}

Termos para indexação: Manejo florestal sustentado, floresta ombrófila mista, Araucaria angustifolia.

\section{Araucaria angustifolia management proposal to use the quotient of Liocourt and analysis of increase in a rural property in Lages, Santa Catarina State, Brazil}

\begin{abstract}
The aim of this study was to calculate the ratio of Liocourt and evaluate the diameter distribution in natural forest with 84 hectares of Araucaria angustifolia serving as a parameter for its management in a farm of 176 hectares, located in White Stone Farm, Lages, Santa Catarina State, Brazil, to achieve the concept of balanced forest. Diameter distribution behaved as expected for native forests, generating a distribution curve in J-shaped, showing that it is a forest inbalanced phase of succession as it has lack of trees in higher diameter classes. The value of the ratio of Liocourt was 1.33, similar to other studies with native forests. By using the ratio of Liocourt would it possible to remove 54 trees per hectare in all classes of frequency, to a desired maximum diameter of $35 \mathrm{~cm}$ with a reduction of $7.4 \mathrm{~m}^{2} \mathrm{ha}^{-1}$ of basal area or $620.8 \mathrm{~m}^{2}$ to the settlement, avoiding the stagnation of the forest and 39 trees per hectare for the maximum diameter of $40 \mathrm{~cm}$ with a reduction of $5.27 \mathrm{~m}^{2} \mathrm{ha}^{-1}$ of basal area or $442.7 \mathrm{~m}^{2}$ for the forest.
\end{abstract}

Index terms: Sustainable forest management, floresta ombrófila mista, Araucaria angustifolia.

\section{Introdução}

A Floresta Ombrófila Mista, também denominada de Mata das Araucárias, é considerada integrante do Bioma Mata Atlântica, Lei 11.428/2006 (Brasil, 2006). Essa apresenta alto índice de exploração, apesar de possuir elevado valor de diversidade. As áreas cobertas por araucária primitivamente ocupavam uma extensão de 7,5 milhões de hectares. Atualmente, no Brasil, estas florestas estão restritas quase totalmente a áreas protegidas, que totalizam cerca de 20 mil hectares (Britez et al., 1992).
Esta fitofisionomia é caracterizada pela elevada dominância da espécie Araucaria angustifolia (Bertol.) Kuntze no dossel superior. Abaixo do dossel, existe um grande número de espécies arbóreas, principalmente pertencentes às famílias Myrtaceae e Lauraceae (Nascimento et al., 2001), como a imbuia (Ocotea porosa (Ness.) Angely), o sassafrás, (Ocotea odorifera Rohwer,) e a canela-lageana (Ocotea pulchella Mart.). Também merece destaque o cedro (Cedrela fissilis Vell.).

A madeira da araucária apresenta boas características físicas e mecânicas, sendo usada nas construções em 
geral, caixotaria, móveis, laminados e vários outros usos (Embrapa, 2009). E como principal produto não madeirável, o pinhão, que constitui a semente da espécie com alto valor nutricional.

Sua área de ocorrência estende-se na região Sul do Brasil, entre as latitudes $24^{\circ}$ e $30^{\circ} \mathrm{S}$, em uma altitude que varia de $500 \mathrm{~m}$ a $1.400 \mathrm{~m}$. Ocorre também em áreas disjuntas no Sudeste brasileiro, entre as latitudes $18^{\circ}$ e $24^{\circ} \mathrm{S}$, em uma altitude que varia de $1.400 \mathrm{~m}$ a $1.800 \mathrm{~m}$. Nos locais de maior altitude da região Sul, esta formação faz parte de um mosaico vegetal formado por florestas e campos naturais (Klein, 1960), estes últimos considerados por alguns autores como vegetação relictual, remanescente de um período climático passado, mais seco e frio, durante o Quaternário (Behling \& Pillar, 2006).

Nos últimos séculos, a exploração de espécies madeiráveis como a araucária, a expansão das cidades e a ocupação do solo para agricultura e pecuária, alteraram a florística e a estrutura dessas florestas. Juntamente com atividades mais recentes como a monocultura em larga escala de Pinus sp., aliado à falta de conhecimento de parâmetros para o manejo de florestas naturais em conjunto com a legislação proibitiva, têm contribuído para a redução, estagnação e baixa diversidade dos remanescentes naturais destas florestas, isolando os fragmentos e submetendo-os a níveis de perturbação diversos.

As propriedades no Município de Lages e grande parte da região serrana de Santa Catarina caracterizamse por geomorfologia de relevo ondulado e fortemente ondulado, predominando na área a Floresta Ombrófila Mista ou floresta com araucária, que é utilizada para extrativismo do pinhão. Além disso, os proprietários utilizam para pastoreio de animais (gado), ocasionando o pisoteio e a interrupção da regeneração de outras espécies acompanhantes da araucária, bem como dela própria, sem contar que o não manejo dessa floresta faz com que ela apresente grande densidade de indivíduos, porém de pequenas dimensões, formando um dossel maciço, com pouca luminosidade e a redução do seu ritmo de crescimento.

A Floresta com Araucária ou Floresta Ombrófila Mista cobria originalmente cerca de $200 \mathrm{mil} \mathrm{km}^{2}$ em todo o Brasil, ocorrendo de forma contínua no Rio Grande do Sul, Santa Catarina e Paraná, internando-se até o sul de Minas Gerais e o Rio de Janeiro (Sanquetta, 2003).

Para Sanquetta (2005), uma forma de conservar os remanescentes da Floresta com Araucária é estimular o manejo em bases sustentáveis, com geração e difusão de tecnologias, já que a proteção integral dos fragmentos florestais por força da lei e da fiscalização por si só não é capaz de manter em quantidade e qualidade o que resta.

Segundo Nutto (2001), o manejo de florestas nativas dominadas por araucárias é muito restritivo para proteger estes últimos remanescentes. Além disso, a proteção via proibição de uso significa uma desvalorização do bem, tendo como consequência, em alguns casos, a perda de interesse na conservação da matéria-prima. Por isso, formas de manejo que consideram o lucro e a conservação, geralmente, são mais eficientes. O autor também acrescenta que para tal forma de manejo se faz necessário maior conhecimento básico visando à colheita de árvores singulares e diâmetros específicos.

De acordo com Mello et al. (2003), para que o manejo florestal da araucária possa ser permitido no futuro, deverão ser realizados estudos técnico-científicos que indiquem formas sustentáveis de se utilizar tais recursos, sem riscos à biodiversidade, sendo necessária a definição do ciclo de corte e também o número de árvores por classe de diâmetro que evolui ao longo do tempo importantes pontos a serem abordados no manejo da floresta.

O manejo florestal deve ser entendido como um elemento decisivo para perpetuar a sobrevivência da Araucaria angustifolia, contribuindo para estimular a regeneração natural, aumentar a taxa de crescimento das árvores remanescentes e diminuir as taxas de mortalidade natural da floresta (Sanquetta, 2008).

Para Finger (2006), a distribuição dos diâmetros é definida como a apresentação de dados agrupados em intervalos de classes segundo seus valores, possibilitando o estudo da floresta em relação à sua estrutura juntamente com os hábitos de crescimento da espécie e as condições ambientais.

As florestas naturais apresentam distribuição diamétrica na forma de J-invertido, caracterizando uma comunidade típica autorregenerante, com maior número de indivíduos nas menores classes de diâmetro, representando a maioria da população. Os vários tipos de florestas apresentam distribuições diamétricas diferentes, tanto em sua amplitude como em sua forma. Por isso, a distribuição de diâmetro é característica importante do estoque em crescimento (Ferreira et al. 1998).

Para Scolforo et al. (1997), a partir da estrutura diamétrica, pode-se utilizar o conceito de floresta balanceada por meio da identificação de classes em que existe déficit ou superávit de árvores. Isso 
consiste na proposta de Liocourt, que descreveu as características de uma floresta normal a partir do estudo de povoamentos irregulares. Liocourt constatou que existia certa proporcionalidade entre o número de árvores por categoria diamétrica sucessiva. Desta forma, deduziu a regra de que em povoamentos irregulares, o número de árvores em relação às classes de diâmetro decresce numa progressão geométrica. Esta relação ficou denominada como quociente de Liocourt, da seguinte forma:

$$
\frac{N_{1}}{N_{2}}=\frac{N_{2}}{N_{3}}=\ldots=\frac{N_{n 1}}{N_{n}}=q
$$

Onde: $\mathrm{N}_{1}$ até $\mathrm{N}_{\mathrm{n}}=$ número de árvores das classes de diâmetro sucessivas de 1 até $n$.

Meyer (1952) introduziu o termo Floresta Balanceada para povoamentos inequiâneos dos EUA, México e Suíça, onde o número de árvores por classe de diâmetro decrescia numa progressão geométrica, sendo representada por uma função de densidade, expressa por:

$N i=K \cdot e^{\text {-adi }}$

Onde: $\mathrm{Ni}=$ densidade para a classe de diâmetro di; di = diâmetro do centro de classe; $\mathrm{K}, \mathrm{a}=$ constantes; $\mathrm{e}$ $=$ base logaritmo neperiano.

O incremento destes povoamentos é a chave do manejo sob o método de seleção (Schneider \& Finger, 2000), pois, em povoamentos irregulares, o corte deve ser exatamente igual ao incremento. Assim, em povoamentos cujas densidades sejam diferentes da normal, o corte deve ajustar-se para que de maneira paulatina obtenha-se o balanceamento da floresta.

No conceito de Liocourt existe uma previsão segundo a qual categorias diamétricas grandes devem ser eliminadas, porque seu incremento está abaixo do ritmo das categorias menores. Sugere que mediante simulação ou experimentação se determine o diâmetro que deve ser mantido na área (critério de madurez ou condição de exploração). A característica deste diâmetro deve permitir que o futuro volume de remoção anual do povoamento resulte no máximo.

Dessa forma, observa-se que mesmo não realizando a retirada de indivíduos, a floresta com araucária corre risco de perda de diversidade, pela forma como é utilizada, comprometendo a sua sobrevivência e seu crescimento. Sendo assim, o presente trabalho foi realizado com o objetivo de conhecer a distribuição diamétrica dessa floresta e a frequência de indivíduos que ocorre em cada classe de diâmetro, e elaborar, com a utilização do quociente de Liocourt, uma proposta de manejo sustentado para as florestas de araucária da região Sul, de maneira teórica, visando posterior debate com órgãos de licenciamento ambiental.

\section{Materiais e métodos}

\section{Caracterização da área}

A área de estudo localiza-se na Fazenda Pedras Brancas em Lages, SC, com área total de 176 ha, sendo que 84 ha abrangem uma região de ocorrência natural de araucária.

A região é classificada, segundo Köeppen, como clima $\mathrm{Cfb}$, temperado constantemente úmido, com verão, sem estação seca. A temperatura média anual variando de $13,8{ }^{\circ} \mathrm{C}$ a $15,8{ }^{\circ} \mathrm{C}$. A precipitação pluviométrica total anual, pode variar de $1.360 \mathrm{~mm}$ a $1.600 \mathrm{~mm}$ e a umidade relativa do ar varia de $80 \%$ a $83 \%$ (Epagri, 2002).

Os solos predominantes da região são associações Cambissolo Álico Tb A húmico, textura muito argilosa, relevo suave ondulado mais solos Litólicos Álicos A húmico, textura argilosa, fase pedregosa, relevo ondulado (substrato efusivas da Formação Serra Geral), ambos campo subtropical e Glei Húmico Álico Tb, textura argilosa, fase campo subtropical de várzea, relevo plano (Epagri, 2002).

\section{Obtenção dos dados e equações utilizadas}

Para obtenção do quociente q' de Liocourt, para a avaliação da estrutura diamétrica e proposição do manejo para conversão em floresta balanceada da Floresta com Araucária, foi utilizado o método de área fixa com parcelas temporárias, sendo os dados coletados de 25 parcelas temporárias de $400 \mathrm{~m}^{2}$, totalizando 1 ha de amostragem, onde foram medidos todos os indivíduos com diâmetro à altura do peito (DAP) a partir de $10 \mathrm{~cm}$. A floresta apresentou 363 árvores por hectare e área basal de $28,28 \mathrm{~m}^{2} \mathrm{ha}^{-1}$. Procedida à coleta, os indivíduos foram divididos em classes diamétricas, com intervalo de classe de $5 \mathrm{~cm}$, do menor ao maior valor de DAP, contando-se o número de indivíduos em cada classe (Tabela 1).

$\mathrm{Na}$ propriedade existiam árvores plaqueteadas de inventário anterior realizado pelo proprietário para obtenção de autorização para corte seletivo de alguns indivíduos. $\mathrm{O}$ inventário foi realizado pelo Ibama em 1999, sendo as árvores então enumeradas e medidas. As mesmas árvores foram remedidas e, pela diferença, foi 
obtido o incremento médio anual em diâmetro para estes indivíduos. Utilizando ajuste de equações do trabalho de Hess (2006), pôde ser calculado o volume, a altura e o incremento médio anual para esses dois parâmetros dendrométricos.

A equação de volume e seus parâmetros estatísticos são:

$\mathrm{v}=0,0071+0,00005 \cdot \mathrm{d}^{2}+0,00003 \cdot \mathrm{d}^{2} \cdot \mathrm{h}+0,00004 \cdot \mathrm{d} \cdot \mathrm{h}^{2}$ - 0,0006.h h $^{2}$ com $\mathrm{R}^{2}$ aj. de 0,99 , erro padrão de 0,0254 , coeficiente de variação de 4,16\% e valor de F de 26277,8 (Hess, 2006).

A equação de altura a ser utilizada e seu parâmetros estatísticos são:

$\ln \mathrm{h}=0,47067+0,6311 * \operatorname{lnd}$, com $\mathrm{R}^{2}$ aj. de 0,94 , erro padrão de 0,1366 , coeficiente de variação de 5,76\% e valor de $\mathrm{F}$ de 2238,5 , demonstrando ser altamente significativa (Hess, 2006).

O tempo de ciclo de corte necessário para recuperação foi calculado por:

$\mathrm{CC}: \mathrm{Vr} / \mathrm{IMAtotal}$

onde: $\mathrm{CC}=$ ciclo de corte em anos para recuperação da floresta; $\mathrm{Vr}=$ volume a retirar pelo método de Liocourt e; $\mathrm{IMA}_{\text {total }}=$ incremento anual total do número de árvores a retirar.

Foram utilizadas equações para desenvolvimento das funções relativas a volume e altura da região com estrutura semelhante, devido à dificuldade de se cortar árvores de araucária mesmo para pesquisa, de acordo com a atual legislação.

Tabela 1. Classes de diâmetro e frequência observada por hectare de Araucaria angustifolia para o inventário realizado.

\begin{tabular}{cc}
\hline Classes de diâmetro & Frequência observada por ha \\
\hline 10 & 55 \\
15 & 54 \\
20 & 48 \\
25 & 32 \\
30 & 35 \\
35 & 42 \\
40 & 36 \\
45 & 25 \\
50 & 12 \\
55 & 13 \\
60 & 7 \\
65 & 3 \\
70 & 1 \\
\hline Total & $\mathbf{3 6 3}$ \\
\hline
\end{tabular}

Determinadas as classes diamétricas, foram ajustados os dados de frequência por classe de diâmetro a partir da função de distribuição de Meyer linearizada:

$$
\ln y_{i}=\beta_{0}+\beta_{1} X_{i}+\varepsilon_{i}
$$

Em que: $\ln y_{i}=\operatorname{logaritmo}$ natural da média da frequência por classe de diâmetro, amplitude de $5 \mathrm{~cm}$, por hectare; $X_{\mathrm{i}}=$ centro de classe de diâmetro; e $\beta_{0}, \beta_{1}=$ coeficientes que expressam a estrutura da vegetação em relação à distribuição dos diâmetros; $\varepsilon_{\mathrm{i}}=$ erro aleatório.

Com base na função de distribuição ajustada, foi obtido o quociente " $q$ " intrínseco da vegetação por meio da seguinte equação:

$$
q=\frac{e^{\left(\beta_{0}+\beta_{1} X_{i}\right)}}{e^{\left(\beta_{0}+\beta_{1} X_{i+1}\right)}}
$$

Onde se utiliza a razão entre as frequências de uma classe de diâmetro qualquer $\left(\mathrm{X}_{\mathrm{i}}\right)$ pela frequência imediatamente acima $\left(\mathrm{X}_{\mathrm{i}+1}\right)$.

\section{Resultados e discussão}

A distribuição diamétrica comportou-se como o esperado para florestas inequiâneas (Figura 1), apresentando curva de distribuição assemelhandose a um J-invertido, caracterizando uma floresta não balanceada, por apresentar déficit de árvores nas maiores classes de diâmetro.

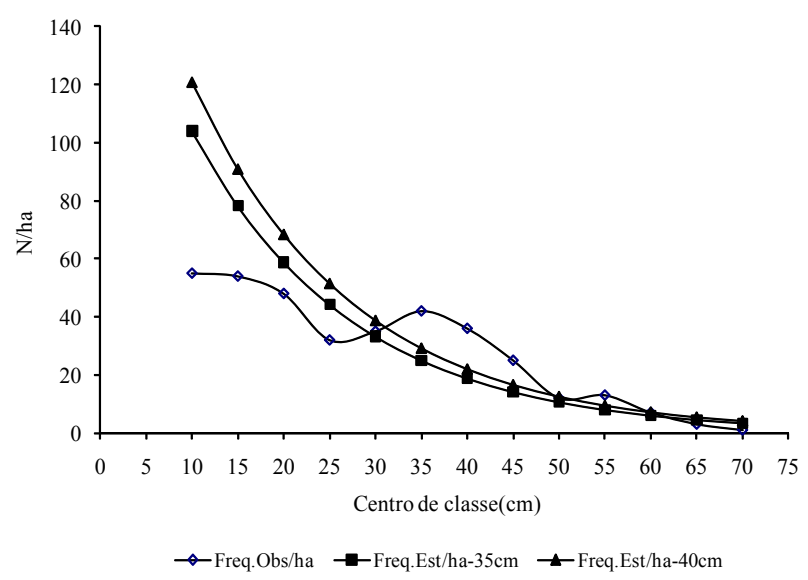

Figura 1. Frequência observada e estimada para os diâmetros máximos desejados de $35 \mathrm{~cm}$ e $40 \mathrm{~cm}$, para Araucaria angustifolia, Município de Lages, SC. 
As distribuições de frequências estimadas originam curvas em J-invertido (Figura 1), porém, com distribuições de frequências balanceadas para todas as classes de diâmetro, o que torna exequível a regulação de corte dessa florestal em longo prazo, estabelecendo-se um regime de manejo sustentado.

Nota-se, ainda, que ocorreram déficit e ausência de árvores nas classes diamétricas para a estrutura balanceada, contudo, o corte permitirá a abertura da floresta e o aumento de incremento diamétrico e volumétrico, bem como a estabilização da vegetação remanescente, como citado em Souza \& Souza (2005), para a Amazônia Oriental.

\section{Alternativa de manejo com base no quociente de Liocourt}

O valor do quociente q' de Liocourt (calculado pela equação 2) para a floresta estudada foi de 1,33, e os coeficientes da equação de distribuição de frequência ajustada e seus parâmetros estatísticos foram $\mathrm{b}_{0}=$ 5,$15272 ; b_{1}=-0,05665 ; R^{2} a j$. de 0,78 ; erro padrão da estimativa de 0,5734 e valor de $\mathrm{F}$ de 44,53.

A maioria dos indivíduos concentrou-se nas classes iniciais de diâmetro e o valor de q' assemelha-se a 1, corroborando com a informação de que esse fragmento de araucária sofreu interferência em sua estrutura diamétrica, em função de intervenções antrópicas anteriores, período em que o corte de araucária era realizado sem planos de manejo.

A alternativa de manejo de Liocourt consiste no estabelecimento de um diâmetro máximo para o manejo. Nesse estudo, estipulou-se o manejo para dois diâmetros máximos desejados, de $35 \mathrm{~cm}$ e de $40 \mathrm{~cm}$. Para o cálculo das frequências estimadas, recalcularam-se os coeficientes da função, sendo para o diâmetro de $35 \mathrm{~cm}$ igual a $\left(b_{0}=5,21491\right.$ e $\left.b_{1}=-0,05704\right)$, e para $40 \mathrm{~cm}$ igual $\mathrm{a}\left(\mathrm{b}_{0}=5,3656\right.$ e $\left.\mathrm{b}_{1}=-0,05704\right)$, podendo-se assim definir a existência de classes superavitárias ou deficitárias em árvores. O número de árvores a ser removido em cada classe diamétrica foi obtido subtraindo-se a frequência observada da estimada (Tabela 2). A tabela também mostra a quantidade de área basal para os indivíduos que podem ser retirados em cada classe diamétrica.

De acordo com a Tabela 2 e o regime de manejo estabelecido, poderão ser removidas árvores das classes de diâmetro $30 \mathrm{~cm}$ a $60 \mathrm{~cm}$ para o diâmetro máximo desejado de $35 \mathrm{~cm}$, representando 54 árvores por hectare e 7,39 $\mathrm{m}^{2}$ ha $^{-1} \mathrm{em}$ área basal, totalizando $620,8 \mathrm{~m}^{2}$ de área basal a serem removidos nos 84 ha.

Para o diâmetro máximo desejado de $40 \mathrm{~cm}$, os valores são apresentados na Tabela 3.

Tabela 2. Frequências observadas e estimadas, número de árvores e área basal a remover para um diâmetro máximo desejado de $35 \mathrm{~cm}$.

\begin{tabular}{|c|c|c|c|c|c|c|}
\hline Centro de classe & Freq.obs ha ${ }^{-1}$ & $\begin{array}{c}\mathbf{G i} \\
\left(\mathbf{m}^{2}\right)\end{array}$ & $\begin{array}{c}G \\
\left(m^{2} h a^{-1}\right)\end{array}$ & Freq.est ha ${ }^{-1}$ & NR & $\begin{array}{c}\mathrm{G} \\
\left(\mathrm{m}^{2} \mathbf{h a}^{-1}\right)\end{array}$ \\
\hline 10 & 55 & 0,00785 & 0,43197 & 104 & - & - \\
\hline 15 & 54 & 0,01767 & 0,95426 & 78 & - & - \\
\hline 20 & 48 & 0,03142 & 1,50796 & 59 & - & - \\
\hline 25 & 32 & 0,04909 & 1,57080 & 44 & - & - \\
\hline 30 & 35 & 0,07069 & 2,47400 & 33 & 2 & 0,12451 \\
\hline 35 & 42 & 0,09621 & 4,04087 & 25 & 17 & 1,63647 \\
\hline 40 & 36 & 0,12566 & 4,52389 & 19 & 17 & 2,16271 \\
\hline 45 & 25 & 0,15904 & 3,97608 & 14 & 11 & 1,72923 \\
\hline 50 & 12 & 0,19635 & 2,35619 & 11 & 1 & 0,27061 \\
\hline 55 & 13 & 0,23758 & 3,08858 & 8 & 5 & 1,19120 \\
\hline 60 & 7 & 0,28274 & 1,97920 & 6 & 1 & 0,28147 \\
\hline 65 & 3 & 0.33183 & 0,99549 & 5 & - & - \\
\hline 70 & 1 & 0.38485 & 0,38485 & 3 & - & - \\
\hline Total & 363 & & 28,28415 & & 54 & 7,39619 \\
\hline
\end{tabular}

Onde: $\mathrm{Gi}$ = área basal em metros quadrados para o centro de classe de diâmetro; $\mathrm{G}=$ área basal em metros quadrados por hectare; NR = número de árvores a remover por centro de classe de diâmetro. 
Tabela 3. Frequências observadas e estimadas, número de árvores e área basal a remover para um diâmetro máximo desejado de $40 \mathrm{~cm}$.

\begin{tabular}{|c|c|c|c|c|c|c|}
\hline Centro de classe & Freq.obs ha ${ }^{-1}$ & $\begin{array}{c}\mathbf{G i} \\
\left(\mathbf{m}^{2}\right)\end{array}$ & $\begin{array}{c}\mathbf{G} \\
\left(\mathbf{m}^{2} \mathbf{h} \mathbf{a}^{-1}\right)\end{array}$ & Freq.est ha $^{-1}$ & NR & $\begin{array}{c}G \\
\left(m^{2} h a^{-1}\right)\end{array}$ \\
\hline 10 & 55 & 0,00785 & 0,43197 & 121 & - & - \\
\hline 15 & 54 & 0,01767 & 0,95426 & 91 & - & - \\
\hline 20 & 48 & 0,03142 & 1,50796 & 68 & - & - \\
\hline 25 & 32 & 0,04909 & 1,57080 & 51 & - & - \\
\hline 30 & 35 & 0,07069 & 2,47400 & 39 & - & - \\
\hline 35 & 42 & 0,09621 & 4,04087 & 29 & 13 & 1,24543 \\
\hline 40 & 36 & 0,12566 & 4,52389 & 22 & 14 & 1,77869 \\
\hline 45 & 25 & 0,15904 & 3,97608 & 16 & 9 & 1,36381 \\
\hline 50 & 12 & 0,19635 & 2,35619 & 12 & - & - \\
\hline 55 & 13 & 0,23758 & 3,08858 & 9 & 4 & 0,88262 \\
\hline 60 & 7 & 0,28274 & 1,97920 & 7 & - & - \\
\hline 65 & 3 & 0.33183 & 0,99549 & 5 & - & - \\
\hline 70 & 1 & 0.38485 & 0,38485 & 4 & - & - \\
\hline Total & 363 & & 28,28415 & & 39 & 5,27055 \\
\hline
\end{tabular}

Onde: $\mathrm{Gi}$ = área basal em metros quadrados para o centro de classe de diâmetro; $\mathrm{G}$ = área basal em metros quadrados por hectare; NR = número de árvores a remover por centro de classe de diâmetro.

Na Tabela 3 verifica-se que podem ser retiradas 39 árvores por hectare, distribuídas nas classes diamétricas de $35,40,45$ e $55 \mathrm{~cm}$ de diâmetro, totalizando $5,27 \mathrm{~m}^{2}$ $\mathrm{ha}^{-1}$ de área basal. Considerando os 84 hectares da área de estudo, $442,7 \mathrm{~m}^{2}$ poderiam ser removidos, conferindo à floresta o conceito de balanceada e evitando a estagnação do povoamento.
O volume total por hectare para o povoamento foi de $255,93 \mathrm{~m}^{3} \mathrm{ha}^{-1}$, representado para os 84 hectares um total de $21.498,12 \mathrm{~m}^{3}$. O uso da equação justifica-se devido às características semelhantes no incremento que esses povoamentos obtiveram com a região estudada por Hess (2006) (Tabela 4). 
Proposta de manejo de Araucaria angustifolia utilizando o quociente de Liocourt e análise de incremento, em propriedade rural no 343 Município de Lages, SC

Tabela 4. Incremento médio anual em diâmetro, altura e volume para Araucaria angustifolia.

\begin{tabular}{|c|c|c|c|c|c|c|c|c|c|}
\hline Árv. no & $\begin{array}{l}\operatorname{dap}_{2} \\
(\mathrm{~cm})\end{array}$ & $\begin{array}{l}\operatorname{dap}_{1} \\
(\mathrm{~cm})\end{array}$ & $\begin{array}{c}\text { IMAd } \\
\left(\mathrm{cm} \mathrm{ano}^{-1}\right)\end{array}$ & $\begin{array}{c}h_{2} \\
(\mathbf{m})\end{array}$ & $\begin{array}{c}h_{1} \\
(\mathbf{m})\end{array}$ & $\begin{array}{c}\text { IMAh } \\
\left(\mathrm{m} \text { ano }^{-1}\right)\end{array}$ & $\mathbf{v}_{2}$ & $\mathrm{v}_{1}$ & $\begin{array}{c}\text { IMAv } \\
\left(\mathrm{m}^{3} \text { ano }^{-1}\right)\end{array}$ \\
\hline 1 & 56,3 & 52,0 & 0,43 & 20,4 & 19,4 & 0,1000 & 2,78908 & 2,27024 & 0,0519 \\
\hline 2 & 61,4 & 59,0 & 0,24 & 21,5 & 21,0 & 0,0535 & 3,48949 & 3,14814 & 0,0341 \\
\hline 3 & 47,0 & 40,0 & 0,70 & 18,2 & 16,4 & 0,1759 & 1,74561 & 1,14508 & 0,0601 \\
\hline 4 & 65,7 & 58,0 & 0,77 & 22,5 & 20,8 & 0,1699 & 4,15480 & 3,01210 & 0,1143 \\
\hline 5 & 45,1 & 40,0 & 0,51 & 17,7 & 16,4 & 0,1292 & 1,56757 & 1,14508 & 0,0422 \\
\hline 6 & 50,0 & 46,0 & 0,40 & 18,9 & 17,9 & 0,0969 & 2,05046 & 1,65047 & 0,0400 \\
\hline 7 & 56,0 & 48,0 & 0,80 & 20,3 & 18,4 & 0,1883 & 2,75079 & 1,84396 & 0,0907 \\
\hline 8 & 56,0 & 52,0 & 0,40 & 20,3 & 19,4 & 0,0928 & 2,75079 & 2,27024 & 0,0481 \\
\hline 9 & 58,5 & 55,0 & 0,35 & 20,9 & 20,1 & 0,0797 & 3,07967 & 2,62544 & 0,0454 \\
\hline 10 & 61,0 & 58,0 & 0,30 & 21,4 & 20,8 & 0,0671 & 3,43113 & 3,01210 & 0,0419 \\
\hline 11 & 47,5 & 47,0 & 0,05 & 18,3 & 18,2 & 0,0122 & 1,79438 & 1,74561 & 0,0049 \\
\hline 12 & 55,5 & 48,0 & 0,75 & 20,2 & 18,4 & 0,1768 & 2,68767 & 1,84396 & 0,0844 \\
\hline 13 & 62,5 & 54,0 & 0,85 & 21,8 & 19,8 & 0,1918 & 3,65306 & 2,50359 & 0,1149 \\
\hline 14 & 52,3 & 48,0 & 0,43 & 19,5 & 18,4 & 0,1025 & 2,30437 & 1,84396 & 0,0460 \\
\hline 15 & 58,8 & 50,0 & 0,88 & 20,9 & 18,9 & 0,2037 & 3,12064 & 2,05046 & 0,1070 \\
\hline 16 & 50,6 & 49,0 & 0,16 & 19,0 & 18,7 & 0,0382 & 2,11498 & 1,94557 & 0,0169 \\
\hline 17 & 51,0 & 45,0 & 0,60 & 19,1 & 17,7 & 0,1454 & 2,15867 & 1,55851 & 0,0600 \\
\hline 18 & 44,7 & 41,0 & 0,37 & 17,6 & 16,7 & 0,0935 & 1,53154 & 1,22171 & 0,0310 \\
\hline 19 & 46,6 & 44,0 & 0,26 & 18,1 & 17,4 & 0,0643 & 1,70717 & 1,46969 & 0,0237 \\
\hline 20 & 59,0 & 55,0 & 0,40 & 21,0 & 20,1 & 0,0910 & 3,14814 & 2,62544 & 0,0523 \\
\hline 21 & 59,5 & 59,0 & 0,05 & 21,1 & 21,0 & 0,0112 & 3,21751 & 3,14814 & 0,0069 \\
\hline 22 & 46,0 & 42,0 & 0,40 & 17,9 & 16,9 & 0,1001 & 1,65047 & 1,30133 & 0,0349 \\
\hline 23 & 64,0 & 61,0 & 0,30 & 22,1 & 21,4 & 0,0659 & 3,88341 & 3,43113 & 0,0452 \\
\hline 24 & 61,0 & 58,0 & 0,30 & 21,4 & 20,8 & 0,0671 & 3,43113 & 3,01210 & 0,0419 \\
\hline 25 & 70,5 & 49,0 & 2,15 & 23,5 & 18,7 & 0,4817 & 4,98157 & 1,94557 & 0,3036 \\
\hline 26 & 47,7 & 47,0 & 0,07 & 18,4 & 18,2 & 0,0170 & 1,81412 & 1,74561 & 0,0069 \\
\hline 27 & 58,0 & 53,0 & 0,50 & 20,8 & 19,6 & 0,1148 & 3,01210 & 2,38520 & 0,0627 \\
\hline 28 & 47,5 & 40,0 & 0,75 & 18,3 & 16,4 & 0,1881 & 1,79438 & 1,14508 & 0,0649 \\
\hline 29 & 44,5 & 44,0 & 0,05 & 17,6 & 17,4 & 0,0125 & 1,51371 & 1,46969 & 0,0044 \\
\hline 30 & 44,0 & 41,0 & 0,30 & 17,4 & 16,7 & 0,0760 & 1,46969 & 1,22171 & 0,0248 \\
\hline 31 & 56,4 & 52,0 & 0,44 & 20,4 & 19,4 & 0,1019 & 2,80191 & 2,27024 & 0,0532 \\
\hline 32 & 47,2 & 41,0 & 0,62 & 18,2 & 16,7 & 0,1550 & 1,76502 & 1,22171 & 0,0543 \\
\hline 33 & 42,9 & 40,0 & 0,29 & 17,2 & 16,4 & 0,0742 & 1,37558 & 1,14508 & 0,0230 \\
\hline 34 & 47,9 & 43,0 & 0,49 & 18,4 & 17,2 & 0,1211 & 1,83398 & 1,38398 & 0,0450 \\
\hline 35 & 47,7 & 40,0 & 0,77 & 18,4 & 16,4 & 0,1930 & 1,81412 & 1,14508 & 0,0669 \\
\hline 36 & 67,3 & 60,0 & 0,73 & 22,8 & 21,2 & 0,1594 & 4,42037 & 3,28780 & 0,1133 \\
\hline 37 & 49,2 & 47,0 & 0,22 & 18,7 & 18,2 & 0,0533 & 1,96628 & 1,74561 & 0,0221 \\
\hline 38 & 80,0 & 75,0 & 0,50 & 25,4 & 24,4 & 0,1015 & 6,89242 & 5,84018 & 0,1052 \\
\hline 39 & 49,0 & 47,0 & 0,20 & 18,7 & 18,2 & 0,0485 & 1,94557 & 1,74561 & 0,0200 \\
\hline 40 & 46,0 & 40,0 & 0,60 & 17,9 & 16,4 & 0,1514 & 1,65047 & 1,14508 & 0,0505 \\
\hline
\end{tabular}

Dap = diâmetro a altura do peito em $\mathrm{cm} ; \mathrm{h}=$ altura em metros; $\mathrm{v}=$ volume em metros cúbicos; IMA = incremento médio anual; (1), (2)= primeira e segunda medição. 
Assim, para a proposição de manejo com diâmetro máximo desejado de $35 \mathrm{~cm}$ e 54 árvores a remover, representou em termos de volume $70,56 \mathrm{~m}^{3} \mathrm{ha}^{-1} \mathrm{e} u \mathrm{um}$ total para a área de $5.927,04 \mathrm{~m}^{3}$, perfazendo assim $27,5 \%$ de intensidade de corte do volume comercial por hectare. Para o diâmetro máximo desejado de $40 \mathrm{~cm}$ e 39 árvores a remover, o volume foi de $49,75 \mathrm{~m}^{3} \mathrm{ha}^{-1}$ e um total de $4.179 \mathrm{~m}^{3}$, equivalendo a $19,4 \%$ de intensidade de corte.

\section{Cálculo do incremento médio anual}

As árvores plaqueteadas foram remedidas neste inventário e, pela diferença das duas medições, obtevese o IMA (Incremento Médio Anual) para os três parâmetros dendrométricos, diâmetro, altura e volume, utilizando os dados de 40 árvores (Tabela 4). O período entre as duas medições foi de 10 anos.

A araucária no Planalto Catarinense apresenta incremento médio anual de valores médios em diâmetro para os 40 dados de $0,48 \mathrm{~cm}^{2} \mathrm{ano}^{-1}$. Para volume, o valor foi de $0,0565 \mathrm{~m}^{3} \mathrm{ano}^{-1}$. A altura apresentou um valor de incremento de $0,11 \mathrm{~m}^{2} \mathrm{ano}^{-1}$ (Tabela 4 ).

Com os dados do incremento médio anual, do número de árvores a retirar e do volume de extração pelo método de Liocourt, pode-se calcular o ciclo de corte, isto é, ciclo de anos para a nova intervenção na floresta. Para esse povoamento, o ciclo é de 23 anos, ou seja, o tempo necessário para a floresta repor o volume retirado. $\mathrm{O}$ cálculo teve como base o incremento médio de 0,0565 $\mathrm{m}^{3}$ ano $^{-1}$, o número de árvores a retirar (54 para o manejo com diâmetro máximo de $35 \mathrm{~cm}$ e 39 árvores para o de $40 \mathrm{~cm})$ e o volume retirado $\left(70,56 \mathrm{~m}^{3}\right.$ e $49,75 \mathrm{~m}^{3}$, respectivamente). Mattos et al. (2007) encontraram um incremento em diâmetro de $0,40 \mathrm{~cm}$ para um período de 10 anos, para a mesma espécie em povoamento natural em Caçador, SC. Hess e Schneider (2010), em estudo de crescimento para araucária em três locais do Rio Grande do Sul, encontrou um incremento diamétrico de $0,96 \mathrm{~cm}$ ano $^{-1}$, em altura de $0,34 \mathrm{~m}$ e em volume de $0,05 \mathrm{~m}^{3}$ ano $^{-1}$.

Há incrementos distintos para o período em consideração (Tabela 4), o qual pode estar associado a algum impedimento rochoso, relevo fortemente ondulado ou características do solo no local do estudo. Nota-se, que de forma geral, todos os parâmetros apresentaram baixo incremento, que pode estar relacionado à falta de condução do povoamento, florestas nativas, exploração desordenada, floresta estagnada, sem intervenção e controle de densidade e/ou características da fertilidade do solo e bióticas do ambiente.

\section{Conclusões}

Conclui-se que o fragmento de floresta com araucária não se encontra balanceado, pois apresenta déficit de árvores em algumas classes de diâmetro. $\mathrm{O}$ fragmento apresentou para a proposta de manejo com diâmetro de $35 \mathrm{~cm}$ e de $40 \mathrm{~cm}$, pelo índice de Liocourt, indivíduos a serem retirados nas classes intermediárias e superiores, comprovando que o povoamento consegue recrutar indivíduos para essas classes de diâmetro.

A distribuição de frequência comportou-se como previsto para florestas inequiâneas, ou seja, na forma de J-invertido. O quociente de Liocourt foi de 1,33, semelhante a estudos já realizados em outras fisionomias florestais. $\mathrm{O}$ valor próximo a 1 representa que a floresta sofreu perturbações anteriores, no caso, exploração comercial de madeira, e que, na atualidade, encontra-se estagnado o crescimento da floresta.

Pela proposição de manejo de Liocourt, poderiam ser retirados $7,4 \mathrm{~m}^{2}$ ha $^{-1}$ de área basal para um diâmetro limite de manejo de $35 \mathrm{~cm} \mathrm{e} 5,27 \mathrm{~m}^{2}$ ha ${ }^{-1}$ para o diâmetro de manejo de $40 \mathrm{~cm}$, totalizando, respectivamente para os 84 hectares do povoamento, a retirada de $620,8 \mathrm{~m}^{2}$ de área basal e volume de 5.927,04 $\mathrm{m}^{3}$ e 442,7 m²de área basal e volume de $4.179 \mathrm{~m}^{3}$, conduzindo a floresta para uma estrutura balanceada.

A análise do incremento, realizada pela diferença de duas medições permitiu calcular o incremento médio anual para os parâmetros dendrométricos de diâmetro, altura e volume. Os valores encontrados demonstram um crescimento lento da espécie, que pode ser decorrentes da condições do relevo, falta de manejo da floresta nativa e controle da densidade da população, principalmente nos períodos iniciais do seu desenvolvimento.

O incremento médio anual em diâmetro foi de $0,48 \mathrm{~cm}$ ano ${ }^{-1}$, em altura de $0,11 \mathrm{~m}^{2} o^{-1}$ e em volume de 0,0565 $\mathrm{m}^{3} \mathrm{ano}^{-1}$ por árvore. Assim, permite-se concluir que as florestas nativas necessitam e possibilitam a elaboração de planos de manejo para sua utilização, com um ciclo de corte de 23 anos, com base no seu incremento e, usando-se um instrumento como a proposição de De Liocourt, melhorando o crescimento das espécies nativas e auxiliando proprietários rurais no melhor do aproveitamento dos recursos naturais e aumento da receita econômica em sua propriedade. 


\section{Referências}

BRASIL. Lei n ${ }^{0} 11.428$, de 22 de dezembro de 2006. Bioma Mata Atlântica. Diário Oficial [da] República Federativa do Brasil, Brasília, DF, 26 dez. 2006.

BEHLING, H.; PILLAR, V. D. Late quaternary vegetation, biodiversity and fire dynamics on the southern brazilian highland and their implication for conservation and management of modern araucaria forest and grassland ecosystems. Philosophical Transactions of the Royal Society B: Biological Sciences, v. 362, p. 243-251, 2006.

BRITEZ, R. M.; REISSMAN, C. B.; SILVA, S.M.; SANTOS FILHO, A. dos. Deposição estacional de serrapilheira e macronutrientes em uma floresta de araucária, São Mateus do Sul, PR. Revista do Instituto Florestal, v. 4, pt. 3, p. 766-772, edição especial, mar., 1992. Edição dos Anais do $2^{\circ}$ Congresso Nacional sobre Essências Nativas, 1992, São Paulo.

EMBRAPA. Cultivo do Pinheiro do Paraná. Disponível em $<$ http://sistemasdeproducao.cnptia.embrapa.br/FontesHTML/ PinheirodoParana/CultivodoPinheirodoParana/index.htm_>. Acesso em: 15 set. 2009.

EPAGRI. Dados e Informações Biofísicas da Unidade de Planejamento Regional Planalto Sul Catarinense - UPR 3, Florianópolis: EPAGRI/CIRAM, 2002, 70 p.

FERREIRA, R. L. C.; SOUZA, A. L.; JESUS, R. M. de. Dinâmica da estrutura de uma floresta secundária de transição. II - Distribuição diamétrica. Revista Árvore, Viçosa, v. 22, n. 3, p. 331-344, 1998.

FINGER, C. A. G. Biometria Florestal. Santa Maria. Universidade Federal de Santa Maria, 2006. 284 p.

HESS, A. F.; SCHNEIDER, P. R. Crescimento em volume de Araucaria angustifólia (Bertol.) Kuntze em três regiões do Rio Grande do Sul, Brasil. Ciência Florestal, v.20, n.1, p.107-122, jan.-mar., 2010.

HESS, A. F. Inter-relações no crescimento de Araucaria angustifolia (Bertol.) Kuntze em diferentes locais do Rio Grande do Sul. 2006. 178 f. Tese (Curso de Doutorado do Programa de Pós-Graduação em Engenharia Florestal) Universidade Federal de Santa Maria, Santa Maria.

KLEIN, R. Aspectos dinâmicos do pinheiro brasileiro. Sellowia, v. 12, n. 12, p. 17-44, 1960.
MATTOS, P. P.; SANTOS, A. T. dos; RIVERA, H.; et al. Crescimento de Araucaria angustifolia na Reserva Florestal Embrapa/Epagri, Caçador, SC. Pesquisa Florestal Brasileira, n. 55, p. 107-114, jul./dez. 2007.

MELLO, A. A.; EISFELD, R. L.; SANQUETTA, C. P. Projeção diamétrica e volumétrica da araucária e espécies associadas no sul do Paraná, usando matriz de transição. Revista Acadêmica Agrárias e Ambientais, Curitiba, v. 1, n. 4, p. 55-66, out./dez. 2003.

MEYER, H. A. Structure, growth, and drain in balanced unevenaged forests. Journal of Forestry, v. 50, n. 2, p. 85-92, 1952.

NASCIMENTO, A. T. R.; LONGHI, S. J.; BRENA, D. A. Estrutura e padrões de distribuição espacial de espécies arbóreas em uma amostra de Floresta Ombrófila Mista em Nova Prata, RS. Ciência Florestal, v. 11, n. 1, p. 105-119, jun./dez. 2001.

NUTTO, L. Manejo do crescimento diamétrico de Araucaria angustifolia (Bert.) O. Ktze. baseado na árvore individual. Ciência Florestal, v.11, p.9-25, 2001.

SANQUETTA, C. R. Crescimento, mortalidade e recrutamento em duas florestas de araucária (Araucaria angustifolia (Bert.) O. Ktze.) no Estado do Paraná, Brasil. Revista de Ciências Exatas e Naturais, v. 5, n.1, jan./jun. 2003.

SANQUETTA, C. R. Perspectivas da recuperação e do manejo sustentável das florestas de araucária. Com Ciência: Revista Eletrônica de Jornalismo Científico, n. 68, ago. 2005. Disponível em: <http://www.comciencia.br/reportagens/2005/08/09.shtml>. Acesso em: ??.

SANQUETTA, C. R. Manual para instalação e medição de parcelas permanentes nos Biomas Mata Atlântica e Pampa. S.I.: RedeMap, Curitiba: Funpar, 2008. 43p.

SCOLFORO, J. R.; OLIVEIRA, A. D. S.; SILVA, S. T. O manejo da vegetação nativa através de corte seletivo. In: CURSO DE

MANEJO FLORESTAL SUSTENTÁVEL, 1. 1997, Curitiba.

Tópicos de manejo florestal sustentável. Colombo: EMBRAPACNPF, 1997. 253 p.

SCHNEIDER, P. R.; FINGER, C. A. G. Manejo Sustentado de Florestas Inequiâneas Heterogêneas. Santa. Maria: UFSM, 2000. $195 \mathrm{p}$

SOUZA, D. R.; SOUZA, A. L. Emprego do método BDq de seleção após a exploração florestal em Floresta Ombrófila Densa de Terra Firme, Amazônia Oriental. Revista Árvore, v. 29, n. 4, p. 617-625, jul./ago. 2005.

Recebido em 30 de junho de 2010 e aprovado em 29 de novembro de 2010 